\title{
Estenosis péptica del esófago: resultados alejados del tratamiento conservador
}

\author{
Mario Anselmi $\mathbf{M}^{\mathbf{1}, 2}$, G erardo 0 rellana $\mathrm{F}^{\mathbf{1}}$, \\ Franco Innocenti $\mathrm{C}^{1}$, Julio Salgado $0^{1}$. \\ Peptic stricture of the esophagus: \\ Long term outcome of conservative \\ treatment
}

Background: Endoscopic dilatation of esophageal strictures is a simple and safe procedure. Aim: To analyze the long term outcome of conservative treatment for esophageal peptic stricture in patients with high surgical risk. Patients and methods: Twenty consecutive patients, 13 male, whose mean age was 75.2 years, with a peptic stricture of the esophagus and high surgical risk were prospectively studied. All were subjected to endoscopic esophageal dilatation and treated with continuous medical antireflux therapy thereafter. Results: Only five patients complied with antireflux treatment on a regular basis. The remaining 15 were non compliant or abandoned it. A total of 56 dilatations were done (mean 2.8 per patient, range 1-6). No complications were observed after the procedure. With a mean follow up period of 49 months, the outcome of the conservative treatment was classified as excellent or good in all the cases. Eight patients (40\%) died of causes unrelated to the treatment. Two patients had an organic foreing body impactation. This situation was solved endoscopically in both. Conclusion: In high risk patients, endoscopic dilatation, with or without regular antireflux medical treatment is a simple, safe and effective therapy in the management of peptic oesophagel stenosis (Rev Méd Chile 2003; 131: 1111-6).

(Key Words: Endoscopy, digestive system; Esophageal stenosis; Gastroesophageal reflux)

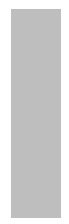

Recibido el 21 de enero, 2003. Aceptado en versión corregida el 12 de agosto, 2003.

Trabajo parcialmente financiado por Dirección de Investigación. Universidad de Concepción. Proyecto Interno \# 96.081.022.

${ }^{1}$ Departamento de Cirugía, Facultad de Medicina, Universidad de Concepción.

${ }^{2}$ Unidad de Cirugía Endoscópica, Servicio de Cirugía, Hospital Guillermo Grant B., Concepción.

Correspondencia a: Dr. Mario Anselmi M.

Fono-Fax 41-204881. E-mail: anselmi@entelchile.net 
$\mathrm{S}_{\mathrm{a}}^{\mathrm{e}}$ estima que la estenosis péptica del esófago aparece entre $1,2 \%$ y $10 \%$ a lo largo de la evolución de la enfermedad en los pacientes que requieren evaluación médica por síntomas de reflujo gastroesofágico ${ }^{1,2}$ y que la mayoría de estos enfermos son de edad avanzada, con compromiso del estado general y patología concomitante ${ }^{3-5}$, lo que hace que el riesgo quirúrgico sea mayor. Por otro lado, la presencia de esta complicación se asocia a resultados quirúrgicos menos satisfactorios que los obtenidos en pacientes con reflujo no complicado 6,7 .

Ahora bien, la dilatación endoscópica del esófago se ha convertido en una técnica simple, segura y efectiva para mantener un lumen adecuado del órgano ${ }^{8}$. Esto, junto a la aparición de potentes fármacos antirreflujo, sugiere que la asociación de ambas terapias puede constituir, en algunos casos, una alternativa útil en el manejo de la estenosis péptica del esófago ${ }^{9,10}$. No existen publicaciones previas en nuestro país al respecto.

El objetivo de este trabajo es analizar, en forma prospectiva, los resultados tardíos del tratamiento conservador de las estenosis pépticas del esófago mediante dilatación en pacientes de alto riesgo quirúrgico.

\section{PACIENTES Y MÉTODO}

Se estudiaron en forma prospectiva 20 pacientes portadores de una estenosis péptica del esófago, que consultaron en la Unidad de Cirugía Endoscópica del Servicio de Cinugía del Hospital Guillermo Grant Benavente de Concepción, entre el 1 de enero de 1994 y el 31 de diciembre de 1997. Se ingresaron al estudio sólo pacientes mayores de 65 años, con patología concomitante que significara alto riesgo quirúrgico. El promedio de edad fue $75,2+9$ años (rango: 65-83 años), 13 (65\%) eran hombres y 7 (35\%) mujeres. En todos los casos se exigió confirmación radiológica y endoscópica de la estenosis y se descartó una neoplasia mediante biopsia.

Desde el punto de vista clínico, se analizó el grado de disfagia inicial de acuerdo a la siguiente clasificación: Leve: sólo para algunos sólidos. Moderada: posibilidad de ingerir sólo semisólidos. Severa: posibilidad de ingerir sólo líquidos.

Al momento del diagnóstico clínico, un enfermo $(5 \%)$ acusaba disfagia leve, en $5(25 \%)$ era

\section{Tabla 1. G rado de severidad de la estenosis (endoscópica)}

\begin{tabular}{|c|c|}
\hline Leve: & $\begin{array}{l}\text { Estenosis evidente del lumen } \\
\text { pero que permite el paso del } \\
\text { endoscopio hacia distal (diámetro } \\
\text { exterior del extremo distal del } \\
\text { instrumento } 10 \mathrm{~mm} \text { ). }\end{array}$ \\
\hline Moderada: & $\begin{array}{l}\text { Se observa lumen distal, pero no } \\
\text { es posible el paso del instrumen- } \\
\text { to ( }>3 \mathrm{~mm} \text { pero }<10 \mathrm{~mm} \text { de } \\
\text { diámetro). }\end{array}$ \\
\hline Severa: & $\begin{array}{l}\text { Puntiforme ( }<3 \mathrm{~mm} \text { de diámetro, } \\
\text { permite sólo el paso de la pinza de } \\
\text { biopsia cerrada o el alambre guía). }\end{array}$ \\
\hline
\end{tabular}

moderada y 14 (70\%) presentaban disfagia severa. De éstos, 4 (20\%) estaban en afagia.

La estenosis estaba ubicada en el esófago distal en todos los casos. Del total, 14 pacientes (70\%) eran portadores de una hernia del hiato por deslizamiento, 4 (20\%) presentaban esófago de Barrett, en 1 (5\%) la estenosis era secundaria a una cardiomiotomía por acalasia y en $2(10 \%)$ no se encontró patología cardioesofágica concomitante.

Endoscópicamente, el grado de estenosis esofágica se clasificó en: leve, moderada y severa (Tabla 1).

Al momento del diagnóstico, 15 pacientes (75\%) presentaban una estenosis severa, ésta era moderada en $4(20 \%)$ y leve sólo en un paciente (5\%).

Asegurado el diagnóstico, los pacientes fueron sometidos a dilatación esofágica, de acuerdo al método de Celestin, según técnica descrita previamente $^{8}$. Los dilatadores de Celestin están compuestos por 2 instrumentos, cada uno de los cuales mide $70 \mathrm{~cm}$ de longitud y tienen un canal central que permite el paso de un alambre guía. El extremo distal tiene un diámetro externo de $4 \mathrm{~mm}$ y éste aumenta en forma gradual en intervalos de $2 \mathrm{~mm}$ hacia el mango del instrumento. El primer dilatador cubre un rango que va entre los $4 \mathrm{y}$ los $12 \mathrm{~mm}$ y el segundo entre los 4 y $18 \mathrm{~mm}$.

Confirmada la indicación de dilatación, se introduce luego por el canal de biopsia del endoscopio un alambre guía, el que se pasa bajo visión directa a través de la estenosis. Su progreso hacia distal se sigue y se comprueba mediante radiología. El alambre debe introducirse por lo 
menos $25 \mathrm{~cm}$ más allá de la estenosis. Con el alambre guía en posición, se remueve el endoscopio y bajo visión radiológica, se pasa el primer dilatador. Este, conducido por el alambre, al alcanzar la estenosis, es empujado a través de ella. La maniobra de retirar el dilatador, se sigue también, radiológicamente, de manera de mantener el alambre en posición. Apenas el extremo distal del dilatador aparece en la boca, un ayudante sujeta el alambre, para prevenir su desplazamiento. Luego, de la misma forma, se introduce el dilatador más grande hasta alcanzar el diámetro máximo que es de $18 \mathrm{~mm}$ (54 Fr). Una vez terminado el procedimiento, cada paciente es reexaminado endoscópicamente para determinar el estado del esófago y descartar complicaciones. Todos los procedimientos se efectuaron con anestesia faríngea, sedación con diazepan iv y en forma ambulatoria. Se intentó introducir ya, durante la primera sesión, el dilatador de mayor diámetro (18 mm ó $54 \mathrm{Fr}$ ).

Logrado el máximo lumen esofágico, los pacientes iniciaron terapia antirreflujo habitual a permanencia. Se indicó medidas generales, tales como bajar de peso, elevar la cabecera de la cama $30 \mathrm{~cm} \mathrm{y}$ se adicionaron, además, medidas específicas como el inicio del estudio, el uso de bloqueadores H2 (Ranitidina 150 mgs c/12 h) y posteriormente, cuando se hicieron disponibles, un bloqueador de bomba de protones (Omeprazole $20 \mathrm{mgs} \mathrm{c} / 12 \mathrm{~h}$ ), antiácidos según disponibilidad hospitalaria, pero generalmente aluminio hidróxido gel 15 cc (900 mg) o 2 comprimidos de 500 mgs $1 / 2$ hora después de las comidas y en la noche.

Los pacientes se controlaron mensualmente en policlínico de cirugía, donde se extendieron las recetas correspondientes y se evaluaron los resultados del tratamiento, de acuerdo a la siguiente pauta (Tabla 2): El control endoscópico se efectuó rutinariamente cada 6 meses o antes, si el caso lo ameritaba. El intervalo entre dilataciones fue determinado por la recidiva de la disfagia o si durante el control endoscópico así se estimó. Se anotó el número de dilataciones y el intervalo entre éstas por pacientes.

Se consideraron como complicaciones del tratamiento tanto las derivadas de la dilatación misma, como aquellas asociadas a reflujo gastroesofágico (aspiración nocturna, hemorragia, úlcera esofágica, neoplasia en caso de Barrett, etc).
Se analizó el resultado de la dilatación inicial, el número de dilataciones por paciente, la adherencia al tratamiento médico, las complicaciones del tratamiento y el resultado tardío tanto subjetivo, como objetivo (endoscópico) del tratamiento.

El estudio terminó al completar un seguimiento mínimo de 3 años o hasta el fallecimiento. En todos los pacientes se obtuvo consentimiento informado respecto al objetivo y método del proyecto. El protocolo fue revisado y aprobado por el Comité de Etica de la Facultad de Medicina de la Universidad de Concepción.

\section{RESULTADOS}

El resultado funcional de la primera dilatación fue calificado como excelente en $18 / 20$ casos (90\%) y bueno en 2 (10\%).

El seguimiento promedio de los pacientes fue de 46 meses, con un rango entre los 9 y 84 meses. Durante el seguimiento, fallecieron 8 pacientes, ninguno de causa relacionada al reflujo gastroesofágico o a las dilataciones esofágicas (Tabla 3).

\section{Tabla 2. Pauta de evaluación de resultados del tratamiento conservador de la estenosis péptica del esófago}

\begin{tabular}{|lll|}
\hline Resultado & $\begin{array}{l}\text { Grado de } \\
\text { Disfagia }\end{array}$ & $\begin{array}{l}\text { Síntomas } \\
\text { de RGEP }\end{array}$ \\
\hline $\begin{array}{l}\text { Excelente } \\
\text { Bueno }\end{array}$ & Sin & Sin \\
Regular & Ocasional & Ocasional \\
Malo & Frecuente & Frecuentes \\
\hline
\end{tabular}

Tabla 3. Causa de muerte durante el seguimiento (en ningún caso ésta estuvo relacionada al RGEP 0 a la dilatación esofágica)

\begin{tabular}{|lc|}
\hline Causa & $\begin{array}{r}\mathrm{n} \text { de Pacientes } \\
(\mathrm{n}=8)\end{array}$ \\
\hline AVC & 4 \\
Infarto miocardio & 2 \\
Neumonía & 1 \\
Desconocida & 1 \\
\hline
\end{tabular}


Sólo un cuarto de los enfermos mantuvo regularmente el tratamiento medicamentoso. El 75\% restante lo siguió en forma irregular o lo abandonó.

En 16 casos, se efectuaron un total de 56 dilataciones (promedio 2,8 dilataciones por paciente). A 5 enfermos (25\%), no fue necesario someterlos a un procedimiento adicional. En 5 (25\%) se efectuaron 2 dilataciones, 4 (20\%) requirieron 3 y los restantes 6 (30\%) debieron ser sometidos a más de 4 procedimientos. No hubo morbilidad ni mortalidad asociada a las dilataciones.

Durante el seguimiento, en 2 pacientes (10\%) se presentó como complicación la impactación de un cuerpo extraño orgánico, en ambos casos fue solucionada endoscópicamente. No hubo mortalidad asociada a la patología esofágica.

Del punto de vista subjetivo, 12 pacientes (60\%) consideraron el resultado como excelente y 8 (40\%) como bueno. Sin embargo, durante los controles endoscópicos, se pesquisó la presencia de esofagitis en 12 casos (60\%).

\section{DisCUSIÓN}

Los factores asociados al desarrollo de una estenosis péptica, aún son comprendidos sólo parcialmente. Se sabe que su desarrollo se asocia, casi siempre, a edad avanzada, valores de reposo del esfínter esofágico inferior en sus niveles más bajos, reflujo severo y de larga evolución, y a trastornos de la motilidad concomitantes, los que son exacerbados por la formación de estenosis transmural ${ }^{9,11}$. Ejemplos extremos, son las estenosis relativamente precoces que ocurren en el reflujo asociado con la esclerodermia y las que siguen al tratamiento quirúrgico de la acalasia ${ }^{11}$.

No siempre existe correlación entre los síntomas que sufre el paciente y la severidad de la esofagitis. Es así que, en el RGEP la disfagia progresiva puede que no constituya un síntoma importante, hasta que no se haya desarrollado la fibrosis. Esto significa que, un número no despreciable de pacientes, pueden presentar una estenosis péptica del esófago sin relatar una historia 0 tener antecedentes de reflujo ${ }^{13}$. Esto se ve, especialmente, en personas de edad avanzada, haciendo sospechar un cuadro maligno.

Ahora bien, en un paciente con estenosis por reflujo ya instalada, los objetivos del tratamiento son: mejorar la disfagia y prevenir un mayor daño del esófago, tan efectiva y seguramente como sea posible. Esto puede hacerse mediante cirugía o utilizando un tratamiento conservador.

En enfermos de bajo riesgo quirúrgico, incluso añosos, sin alteraciones fibróticas de la pared esofágica, la cirugía antirreflujo, tanto convencional como laparoscópica puede ser altamente exitosa y con una mínima morbimortalidad ${ }^{14-16}$. Sin embargo, la tasa de éxito a largo plazo, en pacientes operados por estenosis péptica, fluctúa entre 52 y $90 \%$. La mayor tasa de resultados deficientes del tratamiento quirúrgico de esta patología, comparado con el del reflujo no complicado, se debe, a la combinación de las mayores dificultades técnicas para lograr un control completo de éste y al hecho que, con frecuencia, hay problemas de motilidad asociados ${ }^{13,17-20}$.

En casos no complicados, la fundoplicatura de Nissen, por vía laparoscópica, es considerada en la actualidad como un método efectivo y seguro para el tratamiento de la esofagitis por reflujo ${ }^{14}$. Sin embargo, en pacientes con reflujo gastroesofágico complicado, con una estenosis péptica, este método estaría contraindicado ${ }^{20}$.

Los componentes básicos de un tratamiento no operatorio son la dilatación de la estenosis, el control del reflujo y la búsqueda de cambios neoplásicos que, con frecuencia, se asocian a la estenosis péptica.

La dilatación del esófago es una técnica establecida desde hace mucho tiempo, para el tratamiento de las estenosis benignas y malignas de este órgano ${ }^{7,21-23}$. En estudios previos ${ }^{8,22}$, hemos probado que dilatación endoscópica del esófago mediante bujías es una técnica simple, efectiva, rápida y segura para el tratamiento de las estenosis benignas y malignas del órgano, especialmente si el procedimiento se efectúa bajo control radiológico.

Datos sobre la eficacia a largo plazo de las dilataciones, como terapia única en el tratamiento de las estenosis péptica del esófago, son más bien escasas. En Chile no existen publicaciones previas al respecto. Los resultados de múltiples estudios extranjeros, al igual que los de este trabajo, coinciden en considerar al manejo mediante dilatación endoscópica, como una terapia efectiva en la mayoría de los pacientes y especialmente indicada en enfermos añosos de alto riesgo quirúrgico ${ }^{24,25}$. 
Los buenos resultados a largo plazo del tratamiento conservador fluctúan entre 64 y $96 \% 5,9,21,26$, que son comparables con los obtenidos con el tratamiento quirúrgico ${ }^{5,21}$. Tampoco hay diferencia en cuanto al grado de satisfacción del paciente con ambas terapias ${ }^{27}$. Sin embargo, la diferencia entre las dos modalidades de tratamiento reside en la mortalidad operatoria, especialmente en pacientes añosos ${ }^{5,21,28}$. En nuestra serie no hubo mortalidad asociada al manejo conservador, sin embargo, durante el seguimiento falleció $40 \%$ de los pacientes, como resultado de la patología agregada presente. Por lo tanto, la expectativa de vida parece ser un factor muy importante a considerar en la elección del tratamiento ${ }^{5}$.

Los resultados de los estudios en que la dilatación se ha asociado a terapias médicas antirreflujo permanentes, han demostrado que, a medida que pasa el tiempo, la necesidad de dilataciones adicionales disminuye. Ogilvie ${ }^{29}$ encontró que $40 \%$ de los pacientes tratados con dilataciones y medicamentos antirreflujo no necesitaron más dilataciones, mientras que el $60 \%$ restante, aunque debió ser sometido a múltiples procedimientos, la frecuencia de éstos disminuyó al cabo de 3 años a un promedio de 0,6 dilataciones por paciente. Similares resultados obtuvo Lanza ${ }^{21}$, estudiando un total de 92 pacientes, encontró que $40 \%$ de los con estenosis severa y sólo $17 \%$ de los con estenosis moderada, necesitaron más de un procedimiento. Sin embargo, $75 \%$ del total no requirió más de una dilatación anual. En nuestra serie, en $25 \%$ de los enfermos no fue necesario un procedimiento adicional.

\section{REFERENCIAS}

1. Ben Rejeb M, Bouche O, Zeitoun P. Study of 47 consecutive patients with esophageal stricture compared with 3880 cases of reflux esophagitis. Dig Dis Sci 1992; 37: 733-6.

2. CARR S, Wicks A. Management of peptic oesophageal stricture. Dig Dis 1993; 11: 197-205.

3. Gainsborough N, Powel-Jackson P. Peptic oesophageal stricture: an age related problem? Postgrad Med J 1992; 68: 571-4.

4. RiCHTER JE. Gatroesophageal reflux disease in the older patient: presentation, treatment and complications. Am J Gastroenterol 2000; 95: 368-73.
Uno de los avances más significativos en el tratamiento de la estenosis péptica del esófago, ha sido la demostración de la utilidad de la supresión ácida como terapia adyuvante para la dilatación de las estenosis ${ }^{30}$. En efecto, en todos los estudios, la terapia antirreflujo ha sido mantenida a permanencia, siendo los fracasos atribuidos, por lo menos en parte, a la suspensión de los medicamentos ${ }^{21}$. Sin embargo, algunos han puesto en duda la significancia clínica del uso de bloqueadores H2 por largo tiempo, en pacientes sometidos a dilatación esofágica $^{31,32}$. Los resultados del presente trabajo tienden a confirmar estos hallazgos. En efecto, en nuestra serie, la adherencia al tratamiento medicamentoso con estos fármacos, se obtuvo sólo en 5/ 20 pacientes (25\%) y aunque el resto lo siguió en forma irregular, el resultado final fue ampliamente satisfactorio. Sin embargo, en los controles, $60 \%$ de los casos presentaba evidencias endoscópicas de esofagitis. Lo anterior enfatiza el evidente beneficio de una terapia medicamentosa antirreflujo permanente, de la que careció en forma regular $75 \%$ de nuestros pacientes, la mayoría de ellos de procedencia rural.

En casos de estenosis pépticas dilatadas, pero resistente a los bloqueadores $\mathrm{H} 2$, el uso de omeprazole facilitó la resolución completa de la estenosis a las 4 y 8 semanas en 30 y $70 \%$ respectivamente y en el porcentaje restante, se obtuvieron buenos resultados, perpetuando el tratamiento con omeprazole ${ }^{33-35}$. La inhibición más prolongada y más intensa de la secreción de ácido, parece ser efectiva, incluso en los más severos casos de esofagitis por reflujo.

5. Wesdorp I, Bartelsman F, den Hartog J, Huibregte K, TүтGAT G. Results of conservative treatment of benign esophageal strictures: a follow up study in 100 patients. Gastroenterology 1982; 82: 48793.

6. Hill L, Gelfand M, Bauermeister D. Simplified management of reflux esophagitis with stricture. Ann Surg 1970; 172: 638-46.

7. HeNDERSON R, MarRYATt G. Reccurrent hiatal hernia: Management by thoracoabdominal total fundoplication gastrosplasty. Can J Surg 1981; 24: 151-3.

8. ANSELMI M. Estenosis benigna del esófago: dilatación mediante el método de Celestin. Rev Chil Cir 1993; 45: 326-31. 
9. Marks RD, Richter JE. Peptic stricture of the esophagus. Am J Gastroenterol 1993; 88: 1160-73.

10. Silvis S, Farahmand M, Johnson J, Ansel $H$, Ho S. A randomized blinded comparison of omeprazole and ranitidine in the treatment of chronic esophageal stricture secondary to acid peptic esophagitis. Gastrointest Endosc 1996; 43: 216-21.

11. Ferriow E, Oliveira R, Matsuda N, Braga F, Dantas R. Aging, esophageal motility, and gastroesophageal reflux disease. J Am Geriatr Soc 1998; 46: 1534-7.

12. Pearson FG. Surgical treatment of peptic strictures. En: Stipa S, Belsey RHR, Moraldi A, eds. Medical and Surgical Problems of the Esophagus. New York. Academic Press, 1981; 159-64.

13. WATSON A. The role of anti-reflux surgery combined with fiberoptic endoscopic dilatation in peptic oesophageal stricture. Am J Surg 1984; 148: 346-9.

14. Bammer T, Hinder R, Kiaus A, Kungier P. Five to eight years outcome of the first laparoscopic Nissen fundoplication. J Gastrointest Surg 2001; 5: 42-8.

15. Aluen R, Rappaport W, Hixson L, Samplner R, Case T, FenNeRTy B. Referral patterns and the results of antireflux operations in patients more than sixty years of age. Surg Gynecol Obstet 1991; 173: 359-62.

16. Trus T, LAYcock W, Wo J. Laparoscopic antireflux surgery in the elderly. Am J Gastroentel 1998; 93: 351-3.

17. SPECHLER S. Complications of gastroesophageal reflux disease. En: Castell DO, ed. The Esophagus, 1st edn. Boston: Little, Brown and Company 1992; 543-56.

18. Henderson R, Pearson F. Preoperative assessment of esophageal pathology. J Thorac Cardiovasc Surg 1976; 72: 512-6.

19. Beggs F, Salama F, Knowies K. Management of benign oesophageal stricture by total fundoplication gastroplasty. JR Coll Surg Edinb 1995; 40: 305-7.

20. Bonavina L, Segalin A, Fumagali U, Peracchia A. Surgical management of benign stricture from reflux oesophagitis. Annal Chir Gyn 1995; 83: 175-8.

21. Lanza F, Graham D. Bougienage is effective therapy for most benign esophageal strictures. JAMA 1978; 240: 844-7.

22. ANSELMI M, SiLVA I. Dilatación endoscópica como método de paliación de las estenosis malignas del esófago y del cardias. Rev Chil Cir 1995; 47: 524-9.

23. Puestow K. Conservative treatment of stenosing disease of the oesophagus. Postgrad Med 1955; 18: 6-14.
24. Patterson D, Graham D, Lacey J, Schwartz J, Alpert E, LanzA F, CAIN G. Natural History of Benign Esophageal stricture treated by dilatation. Gastroenterology 1983; 85: 346-50.

25. Tygat G. Dilation therapy of benign esophageal stenoses. World J Surg 1989; 13: 142-8.

26. Kozarek R. Gastrointestinal dilation. En: Yamada T, Alpers D, Owyang C, Powell D, Silverstein F. Textbook of Gastroenterology 2nd ed. Philadelphia: JB Lippincott Company 1995; 2954-63.

27. JAFFRAY B, ANDERSON J. A patient's perspective on the management of peptic esophageal stricture: experience and results in 113 consecutive patients. Dis Esophagus 1998; 11: 109-13.

28. Hands L, Dennison A, Papavramidis S, Mcintyre R, Bishop $\mathrm{H}$, KetTlewell M. The natural history of peptic oesophageal stricture treated by dilation and anti-reflux therapy alone. Ann R Coll Surg Engl 1989; 71: 306-9.

29. Ogilvie A, Ferguson R, Atkinson M. Outlook with conservative treatment of peptic oesophageal stricture. GUT 1980; 21: 23-5.

30. Beck I, Connon J, Lemire S, Thomson A. Canadian consensus conference on the treatment of gastroesophageal reflux disease. J Gastroenterol 1992; 6: 277-89.

31. Ferguson R, Dronfield M, Atkinson M. Cimetidine in treatment of reflux oesophagitis with peptic stricture. Br Med J 1979; 2: 472-4.

32. Farup P, Modalsi $B$, Tholfsen J. Long-term treatment with $300 \mathrm{mg}$ ranitidine once daily after dilatation of peptic oesophageal strictures. Scand J Gastroenterol 1992; 27: 594-8.

33. Smith P, Kerr G, Cockell R, Ross B, Bates C, Brown $P$ ET AL. A comparison of omeprazole and ranitidine in the prevention of recurrence of benign esophageal stricture. Gastroenterology 1994; 107: 1312-18.

34. Marks R, Richter J, Rizzo J, Koen R, Spenney J, Milus T ET AL. Omeprazole versus $\mathrm{H} 2$ receptors antagonists in treating patients with peptic stricture esophagitis. Gastroenterology 1994; 106: 907-15.

35. KoOP H, KatschinsKi M, ARNoLD R. Conservative treatment of florid peptic esophageal stenosis. Complete elimination by dilatation and omeprazole in H2-blockers refractory cases. Med-Klin 1991; 86: 566-8. 